OPEN ACCESS

Check for updates

\title{
Association of spontaneous abortion with all cause and cause specific premature mortality: prospective cohort study
}

\author{
Yi-Xin Wang, ${ }^{1}$ Lidia Mínguez-Alarcón, ${ }^{2,3}$ Audrey J Gaskins, ${ }^{4}$ Stacey A Missmer, \\ Janet W Rich-Edwards, ${ }^{6,7}$ JoAnn E Manson, ${ }^{2,6,7}$ An Pan, ${ }^{8}$ Jorge E Chavarro ${ }^{1,2,6}$
}

For numbered affiliations see end of the article.

Correspondence to: J E Chavarro jchavarr@hsph.harvard.edu (ORCID 0000-0002-4436-9630) Additional material is published online only. To view please visit the journal online.

Cite this as: $B M J$ 2021;372:n530 http://dx.doi.org/10.1136/bmj.n530 Accepted: 10 February 2021

\section{ABSTRACT}

OBJECTIVE

To investigate the association of spontaneous abortion with the risk of all cause and cause specific premature mortality (death before the age of 70).

DESIGN

Prospective cohort study.

SETTING

The Nurses' Health Study II (1993-2017), United States.

\section{PARTICIPANTS}

101681 ever gravid female nurses participating in the Nurses' Health Study II.

\section{MAIN OUTCOMES MEASURES}

Lifetime occurrence of spontaneous abortion in pregnancies lasting less than 6 months, determined by biennial questionnaires. Hazard ratios and $95 \%$ confidence intervals for all cause and cause specific premature death according to the occurrence of spontaneous abortion, estimated with time dependent Cox proportional hazards models.

RESULTS

During 24 years of follow-up, 2936 premature deaths were recorded, including 1346 deaths from cancer and 269 from cardiovascular disease. Crude all cause mortality rates were comparable for women with and without a history of spontaneous abortion (1.24 per 1000 person years in both groups) but were higher for women experiencing three or more spontaneous abortions (1.47 per 1000 person years) and for women reporting their first spontaneous abortion before the age of 24 (1.69 per 1000 person years). The corresponding age adjusted hazard ratios for all cause premature death during follow-up were 1.02 ( $95 \%$ confidence interval 0.94 to 1.11), 1.39 (1.03 to 1.86 ), and 1.27 (1.11 to 1.46), respectively. After adjusting for confounding factors and updated dietary

\section{WHAT IS ALREADY KNOWN ON THIS TOPIC}

Spontaneous abortion is one of the most common adverse outcomes of pregnancy, with an estimated prevalence of $12-24 \%$

Whether spontaneous abortion is associated with a long term risk of premature death (before the age of 70) is unclear

\section{WHAT THIS STUDY ADDS}

Spontaneous abortion was associated with a greater risk of premature death, particularly from cardiovascular disease

The association was particularly strong for recurrent spontaneous abortions and spontaneous abortions occurring early in a woman's reproductive life

Spontaneous abortion could be an early marker of future health risk in women, including an increased risk of premature death and lifestyle factors, the occurrence of spontaneous abortion was associated with a hazard ratio of 1.19 (95\% confidence interval 1.08 to 1.30 ) for premature mortality during follow-up. The association was stronger for recurrent spontaneous abortions (hazard ratio $1.59,95 \%$ confidence interval 1.17 to 2.15 for three or more spontaneous abortions; 1.23 , 1.00 to 1.50 for two; and $1.16,1.05$ to 1.28 for one compared with none), and for spontaneous abortions occurring early in a woman's reproductive life (1.32, 1.14 to 1.53 for age $\leq 23 ; 1.16,1.01$ to 1.33 for ages 24-29; and 1.12, 0.98 to 1.28 for age $\geq 30$ compared with none). When cause specific mortality was evaluated, the association of spontaneous abortion with premature death was strongest for deaths from cardiovascular disease $(1.48,1.09$ to 1.99). Spontaneous abortion was not related to premature death from cancer (1.08, 0.94 to 1.24$)$.

\section{CONCLUSIONS}

Spontaneous abortion was associated with an increased risk of premature mortality, particularly death from cardiovascular disease.

\section{Introduction}

Reducing a third of premature deaths from noncommunicable diseases by 2030 is a United Nation's sustainable development goal. ${ }^{1}$ Identifying risk factors for death from non-communicable disease to highlight priorities for intervention is needed urgently. Although traditional risk factors that affect both men and women (eg, tobacco use, overweight and obesity, and physical inactivity) explain most of the deaths from noncommunicable diseases globally, ${ }^{2}$ increasing evidence suggests that reproductive factors unique to women are associated with a greater risk of death from noncommunicable diseases. Even if reproductive events do not increase the risk of death from non-communicable diseases, they could be useful as an early stress test of underlying risk factors that cause adverse reproductive outcomes and death from non-communicable diseases. $^{34}$

Spontaneous abortion is one of the most common adverse outcomes of pregnancy, with an estimated prevalence of $12-24 \%$ in clinically recognized pregnancies. ${ }^{5} 6$ Substantial evidence indicates that women with a history of spontaneous abortion have a greater risk of non-communicable diseases, including hypertension, ${ }^{7}$ cardiovascular diseases, ${ }^{7-11}$ and type 2 diabetes. ${ }^{71213}$ Evidence relating spontaneous abortion to mortality, however, is scant and inconsistent. ${ }^{14-16}$ Major weaknesses of previous studies included retrospective assessment of spontaneous abortion in the long distant past, and lack of detailed data on various 
relevant confounders (eg, pre-pregnancy body mass index, history of other pregnancy complications) and mediating lifestyle factors (body mass index, quality of diet, smoking status, and physical activity). Also, whether the association is influenced by the number of spontaneous abortions or age at first spontaneous abortion is unclear. To overcome these limitations, we investigated the associations of spontaneous abortion with all cause and cause specific premature death among participants of the Nurses' Health Study II. The Nurses' Health Study II is an ongoing prospective cohort study of women of reproductive age (aged 2542 at baseline), with continuous follow-up spanning three decades and periodic update of reproductive characteristics and lifestyle and health related factors. ${ }^{17}$

\section{Methods}

\section{Study population}

The Nurses' Health Study II is an ongoing prospective cohort established in 1989 by recruiting 116429 female nurses of reproductive age (25-42 years) living in one of 14 states in the United States. ${ }^{17}$ Participants are followed by biennial questionnaires, which collect information on reproductive history, personal characteristics, lifestyle factors, and the occurrence of diseases. ${ }^{17}$ Participants were eligible for inclusion in the current analysis if they had a pregnancy history at recruitment or reported a pregnancy during followup up to 2009 ( $n=101852)$. We excluded participants who had missing data on date of birth $(n=17)$, refused to report their history of spontaneous abortion $(n=15)$, or who had died before 1993, the baseline year for this analysis $(n=139)$. The total number of women included in our current analysis was 101681.

\section{Determination of reproductive history}

In the 1993 questionnaire, participants reported their lifetime history of pregnancies, and spontaneous and induced abortions, in pregnancies lasting less than six months. Participants also reported age at these events in the age categories: $<18,18-20,21-23$, 24-26, 27-29, 30-34, and $\geq 35$. In each subsequent biennial questionnaire up to 2009, participants reported any new pregnancies, or spontaneous and induced abortions, that occurred during the past two years. In 2009, when most participants had reached menopause, women also reported the outcome and year of all of their pregnancies, including those ending in spontaneous or induced abortion. Self-report of pregnancy loss, including stillbirth and spontaneous abortion, has been validated in other studies; the sensitivity of reporting a loss when one actually occurred was estimated as $75 \% .{ }^{18} 19$ Among women who completed the 2009 questionnaire in this cohort, the proportion of women who reported spontaneous abortion in the biennial questionnaires that were also identified as such in 2009 was $83.7 \%$ (15 558 of 18594). Similarly, the proportion of women without a history of spontaneous abortion in the biennial questionnaires that were also confirmed as such in
2009 was $94.4 \%$ (45953 of 48671 ). To reduce recall bias, we only used spontaneous abortions reported in the biennial questionnaires to determine the occurrence of spontaneous abortions.

\section{Determination of deaths}

Deaths were identified by searching the National Death Index or state vital statistics records, or by reporting from close relatives or postal authorities. Deaths that occurred at ages younger than 70 were defined as premature. $^{2021}$ Causes of death were established by autopsy reports, physician review of medical records, or death certificates, according to the International Classification of Diseases eighth revision (codes for cardiovascular disease and cancer were 390-458 and 140-207, respectively) (supplementary table S1). The validity of this method for determining the number of deaths has been assessed in a similar group of nurses from the Nurses' Health Study. ${ }^{22}$ Briefly, all participants known to be alive from the Massachusetts death records were correctly confirmed by the National Death Index $(n=224)$; of 346 participants known to be dead from the state death records, $96.5 \%$ of the true deaths were correctly identified by the National Death Index.

\section{Assessment of covariates}

A physician's diagnosis of gestational diabetes mellitus and hypertensive disorders of pregnancy were selfreported on the baseline questionnaire and updated every two years up to $2001 .^{2324}$ Race or ethnicity, height, and body weight at age 18 were collected at baseline. Current body weight was collected at baseline and in the follow-up questionnaires. We calculated body mass index before pregnancy and during each follow-up cycle. Marriage status and parental history of myocardial infarction and stroke before the age of 60 were recorded at baseline and updated about every four years. Lifestyle factors, reproductive characteristics, and health related conditions (eg, smoking, menopausal status, use of oral contraceptives, use of aspirin, and pregnancy complications) were collected at baseline and updated every two years. Data on physical activity were recorded every four to six years from 1991. Usual dietary intake, including alcohol consumption, was assessed with a semi-quantitative food frequency questionnaire every four years from 1991. ${ }^{17}$ Participants' overall quality of their diet was evaluated by calculating the Alternative Healthy Eating Index, 2010 version. ${ }^{25}$ The reliability of selfreported gestational diabetes mellitus, hypertensive disorders of pregnancy, smoking habit, body weight, physical activity, and dietary intake has been validated in subgroups of participants from this cohort or similar nurses from the Nurses' Health Study. ${ }^{26-28}$

\section{Data analysis}

Women contributed person time from the date of return of the 1993 questionnaire or follow-up questionnaires when participants reported a pregnancy until death or the end of follow-up (30 June 2017), whichever 
occurred first. Occurrence of spontaneous abortion was updated every two years from 1993 to 2009, and ever gravid participants without spontaneous abortion were regarded as the reference group. Participants were considered exposed to spontaneous abortion from the age of the first report of a spontaneous abortion.

Time dependent multivariable adjusted Cox proportional hazards models were constructed to estimate hazard ratios and 95\% confidence intervals to explore the associations between spontaneous abortion, overall and according to the number of spontaneous abortions and age at first spontaneous abortion, and the risk of all cause and cause specific premature death, while simultaneously adjusting for time varying confounders and risk factors. To control as finely as possible for confounding by age, calendar time, and possible interactions between these two timescales, the models were jointly stratified by age in months (continuous) at the start of follow-up and the calendar year for the current questionnaire cycle. $^{29}$ Multivariable adjusted models included terms for race or ethnicity (white or other) and prepregnancy body mass index $(<25,25-29.9$, or $\geq 30)$, and time varying gravidity $(1,2,3$, or $\geq 4)$, nulliparity (no or yes), marriage status (never $v$ ever), history of gestational diabetes (no or yes), hypertensive disorders of pregnancy (no or yes), menopausal status (premenopausal, postmenopausal, or unsure or biologically uncertain), use of hormone treatment (never, past, or current), daily use of aspirin (no or yes), and parental history of myocardial infarction or stroke (no or yes). In a secondary multivariable model, we further adjusted for time varying lifestyle factors, including current body mass index $(<23,23$ $24.9,25-29.9$, or $\geq 30$ ), smoking status (never, former, current 1-34 cigarettes/day, or current $\geq 35$ cigarettes/ day), physical activity (0, 0.1-1.0, 1.1-2.4, 2.5-5.9, or $\geq 6$ hours/week), and Alternative Healthy Eating Index 2010 diet quality score (five categories). For the covariates with missing values (all $<5 \%$ ), an indicator for missing data was created.

We then tested for effect modification by gravidity, parity, pre-pregnancy body mass index, history of gestational diabetes mellitus and hypertensive disorders of pregnancy, parental history of cardiovascular disease, updated body mass index, quality score for diet, physical activity, and smoking status. Interaction on the multiplicative scale was assessed by conducting likelihood ratio tests and on the additive scale by calculating the relative excess risk caused by interaction. ${ }^{25}$

Several sensitivity analyses were conducted. First, we included never gravid women as a separate category to evaluate whether completing a pregnancy, rather than spontaneously losing a pregnancy, was responsible for the associations seen. Second, we jointly classified participants according to their history of spontaneous and induced abortion to explore whether the interruption of pregnancy, rather than the spontaneous loss of a pregnancy, was responsible for any observed relations. Third, we excluded women who were diagnosed with cancer $(n=2788)$, type 2 diabetes $(n=395)$, or cardiovascular disease $(n=83)$ before 1993. Fourth, the comparison group was restricted to women whose pregnancies ended in a live birth only, by excluding women with other pregnancy outcomes (induced abortion, stillbirth, tubal or ectopic pregnancy) from the comparison group. Fifth, we excluded women who died as a result of complications of pregnancy, childbirth, and the puerperium. Sixth, we excluded women who did not return any followup questionnaires after $1993(\mathrm{n}=976)$. All data were analyzed with SAS 9.4 for UNIX (SAS Institute).

\section{Patient and public involvement}

This research was done without patient involvement. Patients and the public were not invited to comment on the study design and were not consulted to develop patient relevant outcomes or interpret the results. Patients and the public were not invited to contribute to the writing or editing of this document for readability or accuracy.

\section{Results}

Among 101681 gravid women, 25.6\% ( $\mathrm{n}=26102)$ had at least one pregnancy ending in spontaneous abortion. Table 1 shows participants' age standardized characteristics in 1993 according to lifetime history of spontaneous abortion. Women who experienced spontaneous abortion were more likely than women who had not had a spontaneous abortion to use aspirin (2235 of $26102(8.56 \%) v 5195$ of 75579 (6.87\%)), and to have a higher mean gravidity (3.34 (standard deviation 1.71) $v 2.30(1.22)$ ) and higher prevalence of a parental history of myocardial infarction or stroke at baseline (7144 of $26102(27.37 \%) v 19674$ of 75579 (26.03\%)).

During 2367839 person years of follow-up, we reported 2936 premature deaths, including 1346 deaths from cancer and 269 from cardiovascular disease (supplementary table S1). Crude all cause mortality rates were comparable for women with and without a history of spontaneous abortion (1.24 per 1000 person years in both groups, fig 1 ), but were higher for women experiencing three or more spontaneous abortions (1.47 per 1000 person years, table 2 ) and for women reporting their first spontaneous abortion before the age of 24 (1.69 per 1000 person years, table $3)$. The corresponding age adjusted hazard ratios for all cause premature death during follow-up were 1.02 (95\% confidence interval 0.94 to 1.11 ), 1.39 (1.03 to 1.86), and 1.27 (1.11 to 1.46), respectively. Differences in the risk of premature death were more evident after adjustment for gravidity (fig 1, table 2, and table 3) and were stronger after further adjustment for potential confounding factors and post-pregnancy dietary and lifestyle factors, particularly in analyses evaluating the relation between the number of spontaneous abortions (table 2) and age at first spontaneous abortion (table 3).

In the fully adjusted models, women who experienced a spontaneous abortion were 19\% (8-30\%) more 


\begin{tabular}{|c|c|c|}
\hline \multirow[b]{2}{*}{ Characteristic } & \multicolumn{2}{|c|}{ Spontaneous abortion } \\
\hline & No $(n=75579)$ & Yes $(n=26102)$ \\
\hline Age (years; mean (SD))† & $38.85(4.67)$ & $38.32(4.66)$ \\
\hline Gravidity (mean (SD)) & $2.30(1.22)$ & $3.34(1.71)$ \\
\hline Parity (mean (SD)) & $1.88(1.06)$ & $1.97(1.24)$ \\
\hline Pre-pregnancy body mass index (mean (SD)) & $21.08(2.86)$ & $21.06(2.86)$ \\
\hline Total physical activity (hours/week; mean (SD)) & $2.76(4.06)$ & $2.78(4.00)$ \\
\hline Alcohol consumption (g/day; mean (SD)) & $3.03(5.98)$ & $2.96(5.69)$ \\
\hline AHEl-2010 dietary score (mean (SD)) $\ddagger$ & $47.57(10.67)$ & $48.01(10.74)$ \\
\hline Ever or currently married (No (\%)) & $72288(95.65)$ & $25116(96.22)$ \\
\hline History of hypertensive disorders of pregnancy (No (\%)) & $9668(12.79)$ & $3648(13.98)$ \\
\hline History of gestational diabetes (No (\%)) & $3230(4.27)$ & $1369(5.24)$ \\
\hline Aspirin use (No (\%)) & $5195(6.87)$ & $2235(8.56)$ \\
\hline Parental history of myocardial infarction or stroke (No (\%)) & $19674(26.03)$ & $7144(27.37)$ \\
\hline \multicolumn{3}{|l|}{ Race or ethnicity (No (\%)) } \\
\hline Non-Hispanic white & $69112(91.44)$ & $24143(92.49)$ \\
\hline Non-Hispanic black & $1427(1.89)$ & $382(1.46)$ \\
\hline Hispanic & $1399(1.85)$ & $478(1.83)$ \\
\hline Other & $3641(4.82)$ & $1099(4.22)$ \\
\hline \multicolumn{3}{|l|}{ Current body mass index (No (\%)) } \\
\hline$<25$ & $46124(61.03)$ & $16229(62.18)$ \\
\hline $25-29.9$ & $17623(23.32)$ & $5798(22.21)$ \\
\hline$\geq 30$ & $11832(15.65)$ & $4075(15.61)$ \\
\hline \multicolumn{3}{|l|}{ Smoking status (No (\%))§ } \\
\hline Never & $48475(64.75)$ & $16856(64.74)$ \\
\hline Past & $17723(23.67)$ & $6295(24.18)$ \\
\hline Current & $8663(11.58)$ & $2885(1.08)$ \\
\hline $\begin{array}{l}\text { SD=standard deviation; AHEI-2010= Alternative Healthy Eating Ind } \\
\text { *Values are standardized to the age distribution of the study popul } \\
\text { tValue is not age adjusted. } \\
\text { fDietary score in } 1991 \text { was reported. } \\
\text { \$784 women had missing data for smoking status. }\end{array}$ & & \\
\hline
\end{tabular}

likely to die prematurely than women who did not have a spontaneous abortion (fig 1). The multivariable adjusted hazard ratios for all cause premature death according to the number of spontaneous abortions were 1.59 (95\% confidence interval 1.17 to 2.15 ) for three or more spontaneous abortions, 1.23 (1.00 to 1.50) for two spontaneous abortions, and 1.16 (1.05 to 1.28) for one spontaneous abortion relative to none (table 2). The multivariable adjusted hazard ratios for all cause premature mortality according to age at first spontaneous abortion were 1.32 (1.14 to 1.53) for women aged 23 or younger, 1.16 (1.01 to 1.33) for ages 24-29, and 1.12 (0.98 to 1.28 ) for age 30 or older relative to none (table 3 ). We found no evidence of effect modification of the relation between spontaneous abortion and premature death by reproductive characteristics, lifestyle factors, or body mass index (supplementary table S2).

In cause specific mortality analyses, spontaneous abortion was not related to death from cancer (hazard ratio $1.08,95 \%$ confidence interval 0.94 to 1.24 ) but was associated with a greater risk of premature death from cardiovascular disease $(1.48,1.09$ to 1.99$)$ and of all other causes of death combined (1.25, 1.09 to 1.44) (fig 1). These relations were also stronger in women who had a greater number of spontaneous abortions and those who first experienced a spontaneous abortion early in their reproductive life (table 2 and table 3). In analyses where less common causes of death were disaggregated, spontaneous abortion was associated with a greater risk of death attributed to diseases of the gastrointestinal system $(1.97,1.09$ to 3.56) but not related to all other causes of death (table 4).

We then conducted a series of sensitivity analyses to evaluate the robustness of the findings and the possibility that the associations seen were not caused by spontaneous abortion itself. First, we performed an analysis where nulligravid women were included as a separate group to examine whether completing pregnancy was beneficial for mortality from cardiovascular disease rather than spontaneous abortion being detrimental. Wefound that nulligravidity was not related to death from cardiovascular disease in this analysis. Compared with gravid women who had never had a spontaneous abortion, the multivariable adjusted hazard ratio for premature death from cardiovascular disease was 1.47 (95\% confidence interval 1.08 to 1.99 ) for gravid women with a history of spontaneous abortion and 1.21 (0.76 to 1.93) for nulligravid women. Second, to examine whether the termination of a pregnancy rather than spontaneous loss of a pregnancy was responsible for the association with death from cardiovascular disease, we conducted an analysis where induced abortion was classified as a separate comparison group. This analysis showed no relation between induced abortion and mortality from cardiovascular disease. Compared with gravid women who had never had a spontaneous or induced abortion, the multivariable adjusted hazard ratio for 


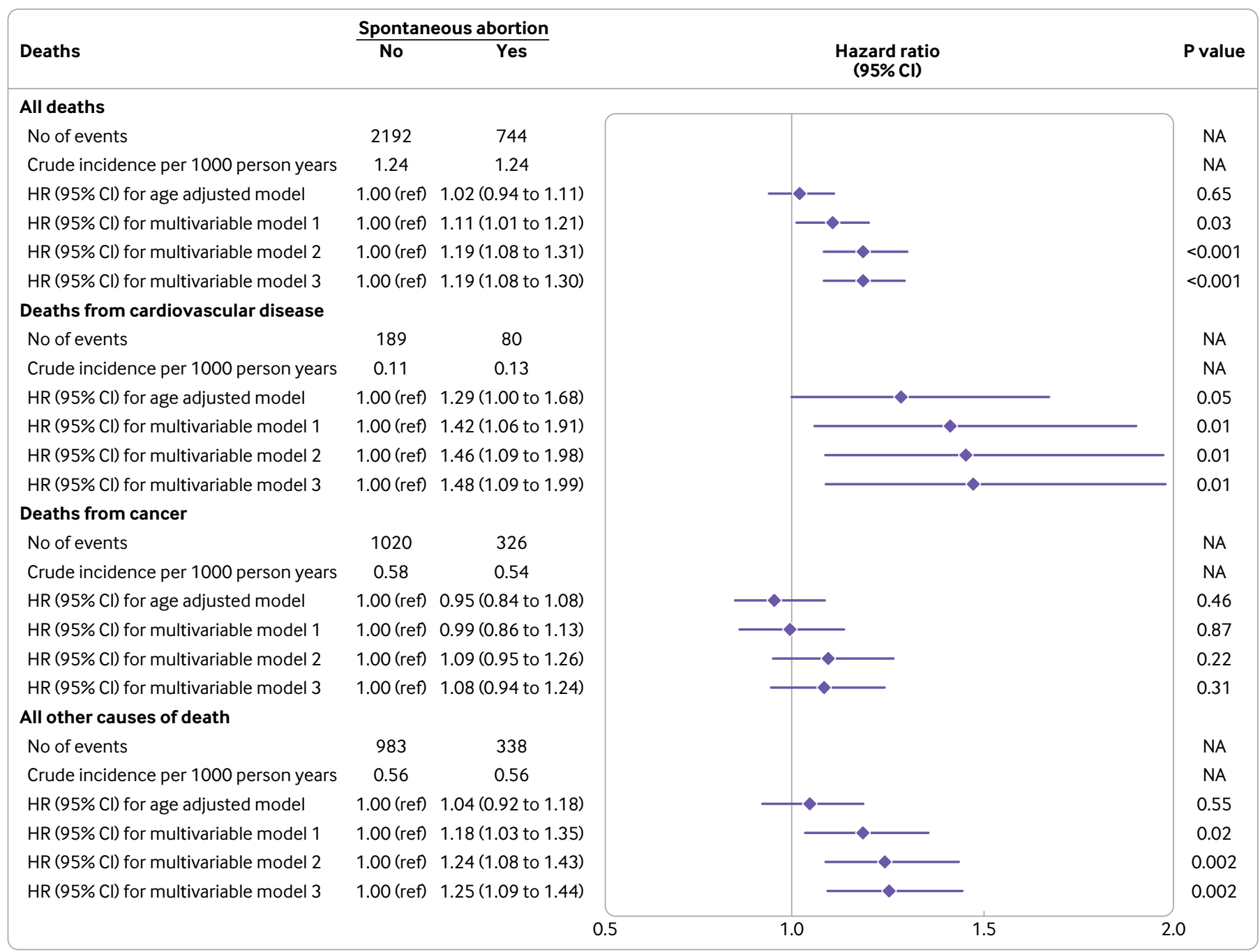

Fig 1 | Adjusted hazard ratios and $95 \%$ confidence intervals for the risk of all cause and cause specific premature death (before age 70 ) according to the overall occurrence of spontaneous abortion in 101681 women (Nurses' Health Study II, 1993-2017). In the age adjusted model, age in months (continuous) at the start of follow-up and calendar year of the current questionnaire cycle were included as stratification variables. Multivariable model 1 was further adjusted for time varying gravidity $(1,2,3$, or $\geq 4)$. Multivariable model 2 was further adjusted for white race or ethnicity (no or yes) and pre-pregnancy body mass index $(<25,25-29.9$, or $\geq 30)$, and time varying nulliparity (no or yes), marriage status (never $v$ ever or currently married), history of gestational diabetes (no or yes), hypertensive disorders of pregnancy (no or yes), menopausal status (premenopausal, postmenopausal, or unsure or biologically uncertain), use of hormone treatment (never, past, or current), daily use of aspirin (no or yes), and parental history of myocardial infarction or stroke (no or yes). Multivariable model 3 was further adjusted for time varying current body mass index ( $23,23-24.9,25-29.9$, or $\geq 30$ ), smoking status (never, former, current 1-34 cigarettes/day, or current $\geq 35$ cigarettes/day), physical activity (0, 0.1 1.0, 1.1-2.4, 2.5-5.9, or $\geq 6$ hours/week), and Alternative Healthy Eating Index 2010 dietary score (five categories). NA=not available

premature death from cardiovascular disease was 1.59 (95\% confidence interval 1.15 to 2.20 ) for women who ever had a spontaneous abortion but no induced abortions, 1.06 (0.70 to 1.62) for women who had ever had an induced abortion but no spontaneous abortions, and 1.01 (0.51 to 2.00) for women who had both spontaneous and induced abortions. The results of associations between spontaneous abortion and premature death were also substantially unchanged when we excluded women who received a diagnosis of cancer, type 2 diabetes, or cardiovascular disease before 1993, when the comparison group was restricted to women whose pregnancies ended in a live birth only, when we excluded deaths caused by complications of pregnancy, childbirth, and the puerperium, and when we excluded women who never returned follow-up questionnaires (supplementary table S3).

\section{Discussion}

Principal findings

The results from this large longitudinal study, with a 24 year follow-up, indicated that spontaneous abortion was associated with a greater risk of all cause premature death, particularly for recurrent spontaneous abortions and spontaneous abortions occurring early in a woman's reproductive life. These associations were primarily because of an increased risk of death from cardiovascular disease, were independent of several shared risk factors (eg, body mass index, smoking, diet, and physical activity), 


\begin{tabular}{|c|c|c|c|c|c|c|}
\hline \multirow[b]{2}{*}{$\begin{array}{l}\text { No of spontaneous } \\
\text { abortions }\end{array}$} & \multirow[b]{2}{*}{ No of events } & \multirow[b]{2}{*}{$\begin{array}{l}\text { Crude incidence per } \\
1000 \text { person years }\end{array}$} & \multicolumn{4}{|c|}{ Adjusted hazard ratios $(95 \% \mathrm{Cl})$} \\
\hline & & & $\begin{array}{l}\text { Age adjusted } \\
\text { model }^{*}\end{array}$ & $\begin{array}{l}\text { Multivariable } \\
\text { model 1† }\end{array}$ & $\begin{array}{l}\text { Multivariable } \\
\text { model } 2 \ddagger\end{array}$ & $\begin{array}{l}\text { Multivariable } \\
\text { model } 3 \S\end{array}$ \\
\hline \multicolumn{7}{|l|}{ All deaths } \\
\hline None & 2192 & 1.24 & 1.00 (reference) & 1.00 (reference) & 1.00 (reference) & 1.00 (reference) \\
\hline 1 & 584 & 1.24 & $1.00(0.91$ to 1.10$)$ & $1.08(0.98$ to 1.19$)$ & $1.17(1.06$ to 1.29$)$ & 1.16 (1.05 to 1.28$)$ \\
\hline 2 & 114 & 1.17 & $1.02(0.85$ to 1.23$)$ & $1.16(0.95$ to 1.41$)$ & $1.22(1.00$ to 1.49$)$ & $1.23(1.00$ to 1.50$)$ \\
\hline$\geq 3$ & 46 & 1.47 & 1.39 (1.03 to 1.86$)$ & $1.58(1.17$ to 2.14$)$ & 1.61 (1.19 to 2.18$)$ & 1.59 (1.17 to 2.15$)$ \\
\hline P for trend & - & - & 0.24 & 0.003 & $<0.001$ & $<0.001$ \\
\hline \multicolumn{7}{|c|}{ Deaths from cardiovascular disease } \\
\hline None & 189 & 0.11 & 1.00 (reference) & 1.00 (reference) & 1.00 (reference) & 1.00 (reference) \\
\hline 1 & 60 & 0.13 & 1.21 (0.90 to 1.62$)$ & 1.34 (0.97 to 1.83$)$ & 1.39 (1.01 to 1.92$)$ & $1.40(1.02$ to 1.93$)$ \\
\hline 2 & 15 & 0.15 & 1.59 (0.94 to 2.68) & $1.84(1.04$ to 3.24$)$ & $1.78(1.01$ to 3.15$)$ & 1.82 (1.03 to 3.22) \\
\hline$\geq 3$ & 5 & 0.16 & $1.84(0.76$ to 4.49$)$ & 2.16 (0.86 to 5.42$)$ & $2.00(0.79$ to 5.06$)$ & $1.96(0.78$ to 4.96$)$ \\
\hline $\mathrm{P}$ for trend & - & - & 0.02 & 0.006 & 0.007 & 0.006 \\
\hline \multicolumn{7}{|l|}{ Deaths from cancer } \\
\hline None & 1020 & 0.58 & 1.00 (reference) & 1.00 (reference) & 1.00 (reference) & 1.00 (reference) \\
\hline 1 & 262 & 0.56 & $0.96(0.84$ to 1.10$)$ & $0.99(0.85$ to 1.14$)$ & 1.09 (0.94 to 1.26$)$ & $1.07(0.92$ to 1.24$)$ \\
\hline 2 & 52 & 0.53 & $0.99(0.75$ to 1.31$)$ & $1.05(0.78$ to 1.41$)$ & $1.17(0.87$ to 1.58$)$ & $1.16(0.86$ to 1.56$)$ \\
\hline$\geq 3$ & 12 & 0.38 & $0.76(0.43$ to 1.35$)$ & $0.82(0.46$ to 1.45$)$ & $0.90(0.50$ to 1.60$)$ & 0.89 (0.50 to 1.59$)$ \\
\hline P for trend & - & - & 0.39 & 0.80 & 0.32 & 0.41 \\
\hline \multicolumn{7}{|c|}{ All other causes of death } \\
\hline None & 983 & 0.56 & 1.00 (reference) & 1.00 (reference) & 1.00 (reference) & 1.00 (reference) \\
\hline 1 & 262 & 0.56 & $1.00(0.88$ to 1.15$)$ & 1.14 (0.98 to 1.32$)$ & 1.21 (1.04 to 1.40$)$ & $1.22(1.05$ to 1.42$)$ \\
\hline 2 & 47 & 0.48 & 0.94 (0.70 to 1.27$)$ & 1.14 (0.84 to 1.55$)$ & 1.16 (0.85 to 1.59$)$ & 1.19 (0.87 to 1.62$)$ \\
\hline$\geq 3$ & 29 & 0.93 & 1.95 (1.35 to 2.83$)$ & 2.39 (1.62 to 3.51$)$ & 2.28 (1.55 to 3.36$)$ & 2.23 (1.51 to 3.28) \\
\hline $\mathrm{P}$ for trend & - & - & 0.12 & $<0.001$ & $<0.001$ & $<0.001$ \\
\hline \multicolumn{7}{|c|}{$\begin{array}{l}\text { *Age in months (continuous) at the start of follow-up and calendar year of the current questionnaire cycle were included as stratification variables. } \\
\text { tMultivariable model } 1 \text { was further adjusted for time varying gravidity }(1,2,3, \text { or } \geq 4) \text {. } \\
\text { fMultivariable model } 2 \text { was further adjusted for white race or ethnicity (no or yes) and pre-pregnancy body mass index ( } 225,25-29.9 \text {, or } \geq 30 \text { ), and time varying nulliparity (no or yes), marriage } \\
\text { status (never } v \text { ever or currently married), history of gestational diabetes (no or yes), hypertensive disorders of pregnancy (no or yes), menopausal status (premenopausal, postmenopausal, or } \\
\text { unsure or biologically uncertain), use of hormone treatment (never, past, or current), daily use of aspirin (no or yes), and parental history of myocardial infarction or stroke (no or yes). } \\
\text { \$Multivariable model } 3 \text { was further adjusted for time varying current body mass index ( } 23,23-24.9,25-29.9, \text { or } \geq 30 \text { ), smoking status (never, former, current } 1-34 \text { cigarettes/day, or current } \geq 35 \\
\text { cigarettes/day), physical activity }(0,0.1-1.0,1.1-2.4,2.5-5.9, \text { or } \geq 6 \text { hours/week), and Alternative Healthy Eating Index } 2010 \text { dietary score (five categories). }\end{array}$} \\
\hline
\end{tabular}

and persisted in a series of sensitivity analyses aimed at isolating the role of spontaneous pregnancy losses from other factors. These findings highlight the growing literature showing that specific reproductive events and risk of chronic disease are linked over a woman's life course; a better understanding of their associations might lead to insights to prevent adverse reproductive outcomes and premature death.

Underlying mechanisms of the associations seen The association between spontaneous abortion and cardiovascular disease might reflect shared mechanistic pathways that contribute to spontaneous abortion and to the development of cardiovascular disease and ultimately premature death. For instance, insulin resistance, chronic kidney disease, and immune disorders have been linked to both spontaneous abortion and the development of clinical risk factors for cardiovascular disease. ${ }^{30-32}$ Likewise, endothelial dysfunction was related to spontaneous abortion by causing defects of the placenta, ${ }^{33}$ and was also implicated in the pathogenesis of cardiovascular, microvascular, and homeostatic dysfunction. ${ }^{34}$ Some genetic or epigenetic features might also predispose women to both spontaneous abortion and cardiovascular disease, which is supported by the finding that parents of women with recurrent spontaneous abortions have a higher incidence of coronary artery disease. ${ }^{35}$ Also, that spontaneous abortion could trigger a cascade that, in turn, leads to premature death, potentially by interactions with established risk factors for cardiovascular disease, has been hypothesized. ${ }^{36}$ In support of this hypothesis, Wagner and colleagues found that women with a history of recurrent pregnancy loss showed a proinflammatory state, manifested as increased levels of high sensitivity $C$ reactive protein, that has been strongly associated with future risk of cardiovascular disease ${ }^{37}$ Further research is needed to understand the underlying mechanisms linking spontaneous abortion to premature death from cardiovascular disease.

\section{Comparison with other studies}

Previous epidemiological studies have reported an association between a history of spontaneous abortion and a greater risk of cardiovascular disease, ${ }^{7-11}$ 38-41 chronic hypertension, ${ }^{7}$ and type 2 diabetes. ${ }^{71213}$ So far, however, few studies have assessed the association of spontaneous abortion with death. In a Danish register based study ( $n=1001266)$, Coleman and colleagues reported that women who had registered a spontaneous abortion exhibited an odds ratio for mortality of 3.06 (95\% confidence interval 2.19 to 4.28 ) compared with women without a history of abortion. ${ }^{14}$ In contrast, in a Japanese cohort of 54652 women aged 40-79, Yamada and colleagues found that women with a history of self-reported pregnancy loss (stillbirth, spontaneous and induced abortions combined) had a 


\begin{tabular}{|c|c|c|c|c|c|c|}
\hline \multirow[b]{2}{*}{$\begin{array}{l}\text { Age at first } \\
\text { abortion }\end{array}$} & \multirow[b]{2}{*}{ No of events } & \multirow[b]{2}{*}{$\begin{array}{l}\text { Crude incidence } \\
\text { per } 1000 \text { person years }\end{array}$} & \multicolumn{4}{|c|}{ Adjusted hazard ratios $(95 \% \mathrm{Cl})$} \\
\hline & & & $\begin{array}{l}\text { Age adjusted } \\
\text { model }^{*}\end{array}$ & $\begin{array}{l}\text { Multivariable } \\
\text { model } 1 \dagger\end{array}$ & $\begin{array}{l}\text { Multivariable } \\
\text { model } 2 \ddagger\end{array}$ & $\begin{array}{l}\text { Multivariable } \\
\text { model } 3 \S\end{array}$ \\
\hline \multicolumn{7}{|l|}{ All deaths } \\
\hline None & 2192 & 1.24 & 1.00 (reference) & 1.00 (reference) & 1.00 (reference) & 1.00 (reference) \\
\hline$\geq 30$ & 274 & 1.09 & $0.93(0.82$ to 1.05$)$ & $1.00(0.87$ to 1.14$)$ & $1.08(0.95$ to 1.24$)$ & $1.12(0.98$ to 1.28$)$ \\
\hline $24-29$ & 254 & 1.17 & 0.97 (0.85 to 1.10$)$ & $1.06(0.92$ to 1.21$)$ & $1.16(1.01$ to 1.34$)$ & $1.16(1.01$ to 1.33$)$ \\
\hline$\leq 23$ & 216 & 1.69 & 1.27 (1.11 to 1.46$)$ & 1.39 (1.20 to 1.60$)$ & 1.41 (1.21 to 1.63$)$ & 1.32 (1.14 to 1.53$)$ \\
\hline P for trend & - & - & 0.06 & $<0.001$ & $<0.001$ & $<0.001$ \\
\hline \multicolumn{7}{|c|}{ Deaths from cardiovascular disease } \\
\hline None & 189 & 0.11 & 1.00 (reference) & 1.00 (reference) & 1.00 (reference) & 1.00 (reference) \\
\hline 230 & 23 & 0.09 & $0.93(0.60$ to 1.43$)$ & 1.02 (0.65 to 1.60$)$ & 1.07 (0.68 to 1.68$)$ & 1.15 (0.73 to 1.82$)$ \\
\hline $24-29$ & 30 & 0.14 & 1.34 (0.91 to 1.97$)$ & 1.49 (0.99 to 2.25$)$ & $1.60(1.05$ to 2.42$)$ & $1.59(1.05$ to 2.41$)$ \\
\hline$\leq 23$ & 27 & 0.21 & 1.85 (1.23 to 2.77$)$ & 2.04 (1.33 to 3.14$)$ & 1.93 (1.25 to 2.98$)$ & 1.78 (1.15 to 2.76$)$ \\
\hline P for trend & - & - & 0.004 & $<0.001$ & 0.001 & 0.002 \\
\hline \multicolumn{7}{|c|}{ Deaths from cancer } \\
\hline None & 1020 & 0.58 & 1.00 (reference) & 1.00 (reference) & 1.00 (reference) & 1.00 (reference) \\
\hline$\geq 30$ & 145 & 0.58 & 1.05 (0.88 to 1.25$)$ & 1.08 (0.90 to 1.30$)$ & $1.21(1.00$ to 1.45$)$ & $1.20(1.00$ to 1.45$)$ \\
\hline $24-29$ & 102 & 0.47 & 0.82 (0.67 to 1.01$)$ & 0.85 (0.69 to 1.06$)$ & $0.96(0.77$ to 1.19$)$ & 0.95 (0.76 to 1.17$)$ \\
\hline$\leq 23$ & 79 & 0.62 & $1.00(0.79$ to 1.25$)$ & $1.03(0.81$ to 1.31$)$ & $1.10(0.86$ to 1.39$)$ & 1.05 (0.83 to 1.34$)$ \\
\hline P for trend & - & - & 0.34 & 0.65 & 0.54 & 0.75 \\
\hline \multicolumn{7}{|c|}{ All other causes of death } \\
\hline None & 983 & 0.56 & 1.00 (reference) & 1.00 (reference) & 1.00 (reference) & 1.00 (reference) \\
\hline$\geq 30$ & 106 & 0.42 & $0.80(0.65$ to 0.97$)$ & 0.90 (0.73 to 1.10$)$ & $0.96(0.77$ to 1.18$)$ & $1.01(0.82$ to 1.24$)$ \\
\hline $24-29$ & 122 & 0.56 & 1.04 (0.86 to 1.26$)$ & 1.20 (0.99 to 1.47$)$ & $1.32(1.08$ to 1.61$)$ & $1.33(1.09$ to 1.63$)$ \\
\hline$\leq 23$ & 110 & 0.86 & 1.45 (1.19 to 1.77$)$ & 1.66 (1.35 to 2.05$)$ & 1.64 (1.33 to 2.02$)$ & 1.53 (1.24 to 1.89$)$ \\
\hline $\mathrm{P}$ for trend & - & - & 0.01 & $<0.001$ & $<0.001$ & $<0.001$ \\
\hline \multicolumn{7}{|c|}{ 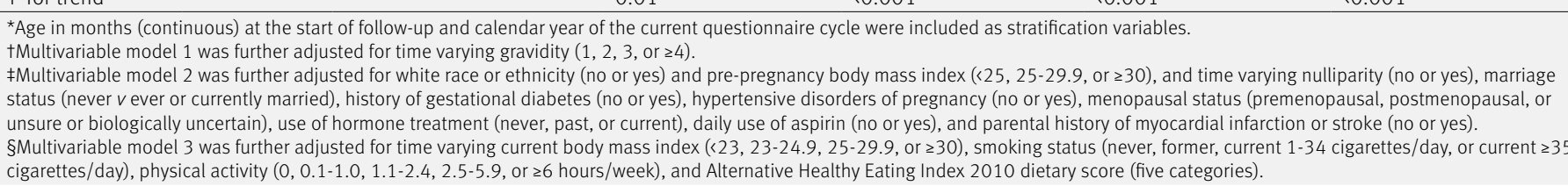 } \\
\hline
\end{tabular}

lower risk of death from cardiovascular disease. ${ }^{15}$ In a Chinese cohort of 267400 female textile workers, ranging in age at enrolment from 30 to over 60, self-reported spontaneous abortion was not related to death from cardiovascular disease. ${ }^{16}$ The major methodological weakness of these previous studies is that they determined the occurrence of spontaneous abortions by retrospective recall at one time point in the long distant past or by hospital register databases, which could result in misclassification because most spontaneous abortions do not result in hospital admission. Also, lack of detailed data on various relevant confounders (eg, gravidity, pre-pregnancy body mass index) and mediating lifestyle factors could lead to residual and unmeasured confounding.

We noticed an increasing trend of a higher risk of premature death with a greater number of spontaneous abortions. The Danish register based study also reported a growing trend of higher mortality rates with an increasing number of spontaneous abortions. ${ }^{14}$ In the Japanese cohort, Yamada and colleagues reported a twofold higher risk of death from ischemic stroke in a young subgroup of participants (aged 40-59) who experienced two or more pregnancy losses than those with no history of pregnancy loss. ${ }^{15}$ Recurrent spontaneous abortion is a major stressful event in a woman's life and has been associated with adverse mental health problems, such as depression and anxiety after the loss, ${ }^{42}$ which are increasingly known as potential risk factors for all cause mortality and mortality related to cardiovascular disease. ${ }^{43}$ The association between spontaneous abortion and all cause premature mortality was also stronger in women who experienced their first spontaneous abortion early in their reproductive life, suggesting that spontaneous abortion at an older age is more likely an indication of age related changes, such as chromosomal abnormalities and decreased ovarian function, ${ }^{44} 45$ rather than an early sign of underlying physiopathology resulting in a greater risk of premature death.

\section{Strengths and limitations}

The strengths of our study include its prospective study design, large sample size, extensive follow-up period across most of the women's reproductive lifespan, and the availability of various reproductive characteristics, and lifestyle and health related factors that allowed us to control for confounding and examine effect modification.

Our study had several limitations. First, misclassification of the occurrence of spontaneous abortions cannot be entirely ruled out, even though self-reported spontaneous abortion has been found to be reliable in this study and in other cohorts. ${ }^{18} 19$ Given our prospective design, however, misclassification should be non-differential with respect to mortality, which would tend to bias effect estimates toward the null. Second, observational studies such as ours can only 


\begin{tabular}{|c|c|c|}
\hline \multirow[b]{2}{*}{ Cause of death* } & \multicolumn{2}{|c|}{ Spontaneous abortion } \\
\hline & No & Yes \\
\hline \multicolumn{3}{|l|}{ Infectious and parasitic diseases } \\
\hline No of events & 47 & 9 \\
\hline Crude incidence per 1000 person years & 0.03 & 0.02 \\
\hline Multivariable adjusted hazard ratio $(95 \% \mathrm{Cl}) \dagger$ & 1.00 (reference) & $0.58(0.27$ to 1.28$)$ \\
\hline \multicolumn{3}{|l|}{ Respiratory disease } \\
\hline No of events & 51 & 27 \\
\hline Crude incidence per 1000 person years & 0.03 & 0.05 \\
\hline Multivariable adjusted hazard ratio $(95 \% \mathrm{Cl}) \dagger$ & 1.00 (reference) & $1.67(0.96$ to 2.91$)$ \\
\hline \multicolumn{3}{|c|}{ Endocrine, nutritional, and metabolic diseases or immunity disorders } \\
\hline No of events & 38 & 10 \\
\hline Crude incidence per 1000 person years & 0.02 & 0.02 \\
\hline Multivariable adjusted hazard ratio $(95 \% \mathrm{Cl}) \dagger$ & 1.00 (reference) & 0.94 (0.43 to 2.08) \\
\hline \multicolumn{3}{|l|}{ Mental disorders } \\
\hline No of events & 34 & 9 \\
\hline Crude incidence per 1000 person years & 0.02 & 0.02 \\
\hline Multivariable adjusted hazard ratio $(95 \% \mathrm{Cl}) \dagger$ & 1.00 (reference) & $0.79(0.35$ to 1.78$)$ \\
\hline \multicolumn{3}{|c|}{ Diseases of the nervous system and sense organs } \\
\hline No of events & 46 & 16 \\
\hline Crude incidence per 1000 person years & 0.03 & 0.03 \\
\hline Multivariable adjusted hazard ratio $(95 \% \mathrm{Cl}) \dagger$ & 1.00 (reference) & $1.59(0.80$ to 3.14$)$ \\
\hline \multicolumn{3}{|c|}{ External causes (eg, accidents, injury, and poisoning) } \\
\hline No of events & 164 & 50 \\
\hline Crude incidence per 1000 person years & 0.09 & 0.08 \\
\hline Multivariable adjusted hazard ratio $(95 \% \mathrm{Cl}) \dagger$ & 1.00 (reference) & $1.09(0.76$ to 1.56$)$ \\
\hline \multicolumn{3}{|l|}{ Suicide } \\
\hline No of events & 81 & 25 \\
\hline Crude incidence per 1000 person years & 0.05 & 0.04 \\
\hline Multivariable adjusted hazard ratio $(95 \% \mathrm{Cl}) \dagger$ & 1.00 (reference) & $1.21(0.73$ to 1.99$)$ \\
\hline \multicolumn{3}{|l|}{ Gastrointestinal diseases } \\
\hline No of events & 44 & 21 \\
\hline Crude incidence per 1000 person years & 0.02 & 0.04 \\
\hline Multivariable adjusted hazard ratio $(95 \% \mathrm{Cl}) \dagger$ & 1.00 (reference) & 1.97 (1.09 to 3.56$)$ \\
\hline \multicolumn{3}{|l|}{ Senility and ill defined diseases } \\
\hline No of events & 111 & 39 \\
\hline Crude incidence per 1000 person years & 0.06 & 0.07 \\
\hline Multivariable adjusted hazard ratio $(95 \% \mathrm{Cl}) \dagger$ & 1.00 (reference) & $1.40(0.92$ to 2.11$)$ \\
\hline \multicolumn{3}{|c|}{$\begin{array}{l}\text { *Only conditions with } 40 \text { or more deaths were included. } \\
\text { tModels were adjusted for white race or ethnicity (no or yes) and pre-pregnancy body mass index ( }(25,25-29.9 \text {, or } \geq 30 \text { ), and time varying age } \\
\text { (continuous), gravidity }(1,2,3, \text { or } \geq 4) \text {, nulliparity (no or yes), marriage status (never } v \text { ever or currently married), history of gestational diabetes (no or } \\
\text { yes), hypertensive disorders of pregnancy (no or yes), menopausal status (premenopausal, postmenopausal, or unsure or biologically uncertain), use of } \\
\text { hormone treatment (never, past, or current), daily use of aspirin (no or yes), parental history of myocardial infarction or stroke (no or yes), current body } \\
\text { mass index ( }(23,23-24.9,25-29.9 \text {, or } \geq 30) \text {, smoking status (never, former, current } 1-34 \text { cigarettes/day, or current } \geq 35 \text { cigarettes/day), physical activity } \\
(0,0.1-1.0,1.1-2.4,2.5-5.9, \text { or } \geq 6 \text { hours/week), and Alternative Healthy Eating Index } 2010 \text { dietary score (five categories). }\end{array}$} \\
\hline
\end{tabular}

demonstrate an association, not causality. Whether experiencing the spontaneous loss of a pregnancy merely unmasks pre-existing risks or instead triggers or accelerates the development of premature death is not known. We found that induced abortion had no association with death from cardiovascular disease, however, suggesting that spontaneous abortion is more likely to be a marker than a cause of premature death. Because randomizing women to experience spontaneous loss of a pregnancy is not possible (or ethical, if it were possible), data from high quality observational studies with extended follow-up are, and are likely to remain, the best possible evidence to look at the association between spontaneous abortion and the risk of premature death. Third, although analyses where induced abortions were considered in a separate comparison group were supportive of our hypothesis, this specific analysis should be interpreted with caution as we are unsure of the validity of self- reports of induced abortions in the United States at the time the data were collected, which might have been influenced by cultural views of induced abortion. Fourth, because most of our cohort was non-Hispanic white women and all participants shared a profession and similar educational qualifications, our findings might not be applicable to other racial or ethnic groups and populations with lower educational attainment. Last, we had only a few cases for many of the causes of death, which limited our power to generate accurate estimates for causes of death other than from all cancers and from all cardiovascular disease combined.

\section{Conclusions}

We found that spontaneous abortion, particularly recurrent spontaneous abortions and spontaneous abortions occurring early in a woman's reproductive life, was associated with an increased risk of premature death. The greater risk of all cause premature mortality 
associated with spontaneous abortion was mainly a result of a higher risk of death from cardiovascular disease. Our results suggest that spontaneous abortion could be an early marker of future health risk in women, including premature death. More research is needed to establish how spontaneous abortion is related to women's long term health and the mechanisms underlying these relations.

\section{AUTHOR AFFILIATIONS}

${ }^{1}$ Department of Nutrition, Harvard T H Chan School of Public Health, 655 Huntington Avenue, Boston, MA 02115, USA

${ }^{2}$ Channing Division of Network Medicine, Department of Medicine, Brigham and Women's Hospital and Harvard Medical School, Boston, MA, USA

${ }^{3}$ Department of Environmental Health, Harvard T H Chan School of Public Health, Boston, MA, USA

${ }^{4}$ Department of Epidemiology, Rollins School of Public Health, Emory University, Atlanta, GA, USA

${ }^{5}$ Department of Obstetrics, Gynecology and Reproductive Biology, College of Human Medicine, Michigan State University, Grand Rapids, MI, USA

${ }^{6}$ Department of Epidemiology, Harvard T H Chan School of Public Health, Boston, MA, USA

${ }^{7}$ Division of Preventive Medicine, Department of Medicine, Brigham and Women's Hospital and Harvard Medical School, Boston, MA, USA

${ }^{8}$ Department of Epidemiology and Biostatistics, School of Public Health, Tongji Medical College, Huazhong University of Science and Technology, Wuhan, Hubei, PR China

We thank the participants and staff of the Nurses' Health Study II for their valuable contributions, and the following state cancer registries for their help: Alabama, Arizona, Arkansas, California, Colorado, Connecticut, Delaware, Florida, Georgia, Idaho, Illinois, Indiana, Iowa, Kentucky, Louisiana, Maine, Maryland, Massachusetts, Michigan, Nebraska, New Hampshire, New Jersey, New York, North Carolina, North Dakota, Ohio, Oklahoma, Oregon, Pennsylvania, Rhode Island, South Carolina, Tennessee, Texas, Virginia, Washington, and Wyoming. The authors assume full responsibility for the analyses and interpretation of these data.

Contributors: Y-XW analyzed and drafted the manuscript. Y-XW and JEC were involved in the study conception and design. LM-A checked the accuracy of the data analysis. Y-XW, LM-A, AJG, SAM, JWR-E, JEM, $A P$, and JEC participated in the interpretation of the results and critical revision of the manuscript. JEC is the study guarantor and accepts full responsibility for the work and conduct of the study, had access to the data, and controlled the decision to publish. The corresponding author attests that all listed authors meet authorship criteria and that no others meeting the criteria have been omitted.

Funding: This study was supported by grants U01-HL145386, U01CA176726, R01-HL034594, and R01-HL088521 from the National Institutes of Health. The funders had no role in considering the study design or in the collection, analysis, interpretation of data, writing of the report, or decision to submit the article for publication.

Competing interests: All authors have completed the ICMJE uniform disclosure form at www.icmje.org/coi_disclosure.pdf and declare: support from the US National Institutes of Health for the submitted work; no financial relationships with any organizations that might have an interest in the submitted work in the previous three years; no other relationships or activities that could appear to have influenced the submitted work.

Ethical approval: The study protocol was approved by the institutional review boards of the Brigham and Women's Hospital and the Harvard T H Chan School of Public Health. The completion of the self-administered questionnaire was considered to imply informed consent. Protocol No: 2009-P-002375.

Data sharing: Data described in the manuscript, code book, and analytic code will not be made publicly available. Further information, including the procedures for obtaining and accessing data from the Nurses' Health Studies II, is described at www.nurseshealthstudy.org/ researchers (email: nhsaccess@channing.harvard.edu).

The lead author (the manuscript's guarantor) affirms that the manuscript is an honest, accurate, and transparent account of the study being reported; that no important aspects of the study have been omitted; and that any discrepancies from the study as planned (and, if relevant, registered) have been explained.

Dissemination to participants and related patient and public communities: Study participants are periodically updated on findings from the study through multiple channels, including a yearly newsletter, the study website, and social media feeds.

Provenance and peer review: Not commissioned; externally peer reviewed.

This is an Open Access article distributed in accordance with the Creative Commons Attribution Non Commercial (CC BY-NC 4.0) license, which permits others to distribute, remix, adapt, build upon this work non-commercially, and license their derivative works on different terms, provided the original work is properly cited and the use is noncommercial. See: http://creativecommons.org/licenses/by-nc/4.0/.

1 Cao B, Bray F, Ilbawi A, Soerjomataram I. Effect on longevity of one-third reduction in premature mortality from non-communicable diseases by 2030: a global analysis of the Sustainable Development Goal health target. Lancet Glob Health 2018;6:e1288-96. doi:10.1016/S2214-109X(18)30411-X

2 Kontis V, Mathers CD, Rehm J, et al. Contribution of six risk factors to achieving the $25 \times 25$ non-communicable disease mortality reduction target: a modelling study. Lancet 2014;384:427-37. doi:10.1016/ S0140-6736(14)60616-4

3 Facca TA, Mastroianni-Kirsztajn G, Sabino ARP, et al. Pregnancy as an early stress test for cardiovascular and kidney disease diagnosis. Pregnancy Hypertens 2018;12:169-73. doi:10.1016/j. preghy.2017.11.008

4 Carbillon L. Pregnancy is an essential spontaneous screening stress test for the risk of early stroke in women. Stroke 2008;39:e138. doi:10.1161/STROKEAHA.107.514190

5 Ammon Avalos L, Galindo C, Li DK. A systematic review to calculate background miscarriage rates using life table analysis. Birth Defects Res A Clin Mol Teratol 2012:94:417-23 doi:10.1002/bdra.23014

6 Robinson GE. Pregnancy loss. Best Pract Res Clin Obstet Gynaecol 2014;28:169-78. doi:10.1016/j.bpobgyn.2013.08.012

7 Horn J, Tanz LJ, Stuart JJ, et al. Early or late pregnancy loss and development of clinical cardiovascular disease risk factors: a prospective cohort study. BJOG 2019;126:33-42. doi:10.1111/1471-0528.15452

8 Smith GC, Pell JP, Walsh D. Spontaneous loss of early pregnancy and risk of ischaemic heart disease in later life: retrospective cohort study. BMJ 2003;326:423-4. doi:10.1136/bmj.326.7386.423

9 Asgharvahedi F, Gholizadeh L, Siabani S. The risk of cardiovascula disease in women with a history of miscarriage and/or stillbirth. Health Care Women Int 2019;40:1117-31. doi:10.1080/07399332 2019.1566332

10 Kharazmi E, Dossus L, Rohrmann S, Kaaks R. Pregnancy loss and risk of cardiovascular disease: a prospective population-based cohort study (EPIC-Heidelberg). Heart 2011;97:49-54. doi:10.1136/ hrt.2010.202226

11 Ranthe MF, Andersen EA, Wohlfahrt J, Bundgaard H, Melbye $\mathrm{M}$, Boyd HA. Pregnancy loss and later risk of atherosclerotic disease. Circulation 2013;127:1775-82. doi:10.1161/ CIRCULATIONAHA.112.000285

12 Liu B, Song L, Li H, et al. History of spontaneous miscarriage and the risk of diabetes mellitus among middle-aged and older Chinese women. Acta Diabetol 2018;55:579-84. doi:10.1007/s00592-018 $1125-z$

13 Kharazmi E, Lukanova A, Teucher B, Groß ML, Kaaks R. Does pregnancy or pregnancy loss increase later maternal risk of diabetes?Eur J Epidemiol 2012;27:357-66. doi:10.1007/s10654012-9683-9

14 Coleman PK, Reardon DC, Calhoun BC. Reproductive history patterns and long-term mortality rates: a Danish, population-based record linkage study. Eur J Public Health 2013;23:569-74. doi:10.1093/ eurpub/cks107

15 Yamada K, Iso H, Cui R, Tamakoshi A. Recurrent Pregnancy Loss and Cardiovascular Disease Mortality in Japanese Women: A Population-Based, Prospective Cohort Study. J Stroke Cerebrovasc Dis 2017;26:1047-54. doi:10.1016/j. jstrokecerebrovasdis.2016.12.018

16 Gallagher LG, Davis LB, Ray RM, et al. Reproductive history and mortality from cardiovascular disease among women textile workers in Shanghai, China. Int J Epidemiol 2011;40:1510-8. doi:10.1093/ ije/dyr134

17 Bao Y, Bertoia ML, Lenart EB, et al. Origin, methods, and evolution of the three Nurses' Health Studies. Am J Public Health 2016;106:1573-81. doi:10.2105/AJPH.2016.303338

18 Kristensen P, Irgens LM. Maternal reproductive history: a registry based comparison of previous pregnancy data derived from maternal recall and data obtained during the actual pregnancy. 
Acta Obstet Gynecol Scand 2000;79:471-7. doi:10.1080/j.16000412.2000.079006471x

19 Wilcox AJ, Horney LF. Accuracy of spontaneous abortion recall. Am J Epidemiol 1984;120:727-33. doi:10.1093/oxfordjournals.aje. a113940

20 Norheim OF, Jha P, Admasu K, et al. Avoiding 40\% of the premature deaths in each country, 2010-30: review of national mortality trends to help quantify the UN sustainable development goal for health. Lancet 2015;385:239-52. doi:10.1016/S01406736(14)61591-9

21 Wang YX, Arvizu M, Rich-Edwards JW, et al. Menstrual cycle regularity and length across the reproductive lifespan and risk of premature mortality: prospective cohort study. BM/ 2020;371:m3464. doi:10.1136/bmj.m3464

22 Rich-Edwards JW, Corsano KA, Stampfer MJ. Test of the National Death Index and Equifax Nationwide Death Search. Am J Epidemiol 1994;140:1016-9. doi:10.1093/oxfordjournals.aje. a117191

23 Solomon CG, Willett WC, Rich-Edwards J, et al. Variability in diagnostic evaluation and criteria for gestational diabetes. Diabetes Care 1996;19:12-6. doi:10.2337/diacare.19.1.12

24 Stuart JJ, Tanz LJ, Missmer SA, et al. Hypertensive disorders of pregnancy and maternal cardiovascular disease risk factor development: an observational cohort study. Ann Intern Med 2018;169:224-32. doi:10.7326/M17-2740

25 Wang YX, Shan Z, Arvizu M, et al. Associations of menstrual cycle characteristics across the reproductive life span and lifestyle factors with risk of type 2 diabetes. JAMA Netw Open 2020;3:e2027928. doi:10.1001/jamanetworkopen.2020.27928

26 Rimm EB, Stampfer MJ, Colditz GA, Chute CG, Litin LB, Willett WC Validity of self-reported waist and hip circumferences in men and women. Epidemiology 1990;1:466-73. doi:10.1097/00001648199011000-00009.

27 Wolf AM, Hunter DJ, Colditz GA, et al. Reproducibility and validity of a self-administered physical activity questionnaire. Int J Epidemiol 1994:23.991-9 doi:10.1093/ije/23.5.991

28 Yuan C, Spiegelman D, Rimm EB, et al. Relative Validity of Nutrient Intakes Assessed by Questionnaire, 24-Hour Recalls, and Diet Records as Compared With Urinary Recovery and Plasma Concentration Biomarkers: Findings for Women. Am Epidemiol 2018;187:1051-63. doi:10.1093/aje/kwx328

29 Wang YX, Arvizu M, Rich-Edwards JW, et al. Hypertensive Disorders of Pregnancy and Subsequent Risk of Premature Mortality. J Am Coll Cardiol 2021;77:1302-12. doi:10.1016/j. jacc.2021.01.018

30 Ahmed SK, Mahmood N, Malalla ZH, Alsobyani FM, Al-Kiyumi IS, Almawi WY. C-reactive protein gene variants associated with recurrent pregnancy loss independent of CRP serum levels: a case-control study. Gene 2015:569:136-40. doi:10.1016/i.gene.2015.05.052

31 Celik N, Evsen MS, Sak ME, Soydinc E, Gul T. Evaluation of the relationship between insulin resistance and recurrent pregnancy loss. Ginekol Pol 2011;82:272-5.
32 Oliver-Williams CT, Heydon EE, Smith GC, Wood AM. Miscarriage and future maternal cardiovascular disease: a systematic review and meta-analysis. Heart 2013;99:1636-44. doi:10.1136/ heartjnl-2012-303237

33 Pasquier E, De Saint Martin L, Bohec C, Collet M, Dignat George F, Mottier D. Unexplained pregnancy loss: a marker of basal endothelia dysfunction?Fertil Steril 2013;100:1013-7. doi:10.1016/j. fertnstert.2013.06.008.

34 Konukoglu D, Uzun H. Endothelial Dysfunction and Hypertension. Adv Exp Med Biol 2017;956:511-40. doi:10.1007/5584_2016_90

35 Smith GC, Wood AM, Pell JP, Hattie J. Recurrent miscarriage is associated with a family history of ischaemic heart disease: a retrospective cohort study. BJOG 2011:118:557-63. doi:10.1111/ j.1471-0528.2010.02890.x

36 Ranthe MF, Boyd HA. Miscarriage and cardiovascular disease. Heart 2015;101:1933-4. doi:10.1136/heartjnl-2015-308383

37 Wagner MM, Jukema JW, Hermes W, et al. Assessment of novel cardiovascular biomarkers in women with a history of recurrent miscarriage. Pregnancy Hypertens 2018;11:129-35. doi:10.1016/j. preghy.2017.10.012

38 Kessous R, Shoham-Vardi I, Pariente G, Sergienko R, Holcberg $\mathrm{G}$, Sheiner E. Recurrent pregnancy loss: a risk factor for long-term maternal atherosclerotic morbidity?Am J Obstet Gynecol 2014;211:414.e1-11. doi:10.1016/j.ajog.2014.05.050

39 Maino A, Siegerink B, Algra A, Martinelli I, Peyvandi F, Rosendaal FR. Pregnancy loss and risk of ischaemic stroke and myocardial infarction. Br J Haematol 2016;174:302-9. doi:10.1111/bjh.14043

40 Parikh NI, Jeppson RP, Berger JS, et al. Reproductive Risk Factors and Coronary Heart Disease in the Women's Health Initiative Observational Study. Circulation 2016;133:2149-58. doi:10.1161/ CIRCULATIONAHA.115.017854

41 Wagner MM, Bhattacharya S, Visser J, Hannaford PC, Bloemenkamp KW. Association between miscarriage and cardiovascular disease in a Scottish cohort. Heart 2015;101:1954-60. doi:10.1136/ heartjnl-2015-307563

42 Kataoka K, Tomiya Y, Sakamoto A, Kamada Y, Hiramatsu Y, Nakatsuka M. Altered autonomic nervous system activity in women with unexplained recurrent pregnancy loss. J Obstet Gynaecol Res 2015;41:912-8. doi:10.1111/jog.12653

43 Meng R, Yu C, Liu N, et al, China Kadoorie Biobank Collaborative Group. Association of depression with allcause and cardiovascular disease mortality among adults in China. JAMA Netw Open 2020;3:e1921043. doi:10.1001/ jamanetworkopen.2019.21043

44 Quinn MM, Cedars MI. Cardiovascular health and ovarian aging. Fertil Steril 2018:110:790-3. doi:10.1016/j.fertnstert.2018.07.1152

45 Wu X, Cai H, Kallianpur A, et al. Impact of premature ovarian failure on mortality and morbidity among Chinese women. PLoS One 2014:9:e89597. doi:10.1371/journal pone.0089597

Web appendix: Supplemental material 